\title{
An Evaluation of the Use of G-CSF as an Adjunct to IVF in Women Who Have Previously Failed Attempts at Pregnancy With IVF
}

\author{
Tasneem Mohamed $^{1^{* \mathbb{D}}}$, Yasmin $_{\text {Adam }^{1}}^{\mathbb{D}}$, Mohamed Iqbal Cassim $^{2}$
}

\begin{abstract}
Objectives: The present study aimed to evaluate the effects of granulocyte colony-stimulating factor (G-CSF) as an adjunct to in vitro fertilization (IVF). In other words, it delved into the influence of G-CSF on the endometrium and the achievement of pregnancy.

Materials and Methods: This retrospective cross-sectional study was conducted on a subgroup of women with two or more previously failed IVFs, who attended a fertility clinic in Johannesburg. These women underwent a procedure of transvaginal infusion of G-CSF in addition to their IVF protocol although endometrial thickness was not a criterion for G-CSF use.

Results: The group included 49 women with a mean age of 38.9 years (SD \pm 6.11 ). The mean number of previous IVFs was 3.1 (SD \pm 1.76$)$. The mean endometrial thickness pre-G-CSF and post-G-CSF was $7.53 \mathrm{~mm}(\mathrm{SD} \pm 2.69)$ and $9.11 \mathrm{~mm}(\mathrm{SD} \pm 2.12)$, respectively. The clinical pregnancy rate was $34.69 \%$. Univariate analysis between the groups of women who achieved or failed to achieve pregnancy showed that the age difference between the two groups was statistically significant $(P=0.0005)$. Further, G-CSF use was associated with increased pregnancy rates in younger women. Finally, although the mean endometrial thickness pre and post-G-CSF were not statistically significant between the two groups $(P>0.05)$, the mean change in endometrial thickness was statistically significant in all women regardless of pregnancy outcome $(P=0.0029)$.

Conclusions: In general, G-CSF is considered as a useful adjunct for the treatment of women with recurrent failed IVFs and aged less than 38 years. Based on the findings, a statistically significant overall expansion of endometrial thickness was reported by using G-CSF. However, it failed to show any association between endometrial expansion and pregnancy outcome.

Keywords: G-CSF, IVF, Endometrium, Pregnancy
\end{abstract}

\section{Introduction}

Pregnancy by standard in vitro fertilisation (IVF) is still not achieved for some infertile woman, even with successful ovulation induction and embryo development. This may be due to defective implantation. A thin endometrium can negatively affect the receptivity of the endometrium to the transferred embryo, leading to its failure for implantation.

Granulocyte Colony-Stimulating Factor in Assisted Reproductive Technology

Granulocyte Colony-stimulating Factor (G-CSF), initially identified as a growth factor for neutrophils, is encoded by a single gene found on chromosome 17 q11-22 (1).

In addition, G-CSF is found to have an important role in human reproduction and is regarded as the basis for studies which combine the use of G-CSF and ART. Further, G-CSF positively affects implantation through its "action on neutrophilic granulocyte proliferation and the macrophages of decidual cells" (2). It is also found to be synthesized de novo in the female reproductive system and hence, it is hypothesized to have some influences on the growth and development of the embryo (2).
Based on the results of a previous study investigating the effect of G-CSF on an embryo culture medium, the inclusion of G-CSF to culture media increased the survival rate of the transferred embryos to week 12 , as well as the live birth rate $(2,3)$.

The Effect of Endometrial Thickness on Fertility Endometrial thickness is an indirect measure of the receptivity of the endometrium. It is thought that the ideal thickness is between $7 \mathrm{~mm}$ and $14 \mathrm{~mm}$ (4).

Richter et al studied the association between endometrial thickness and implantation and found a significant correlation between increasing endometrial thickness and clinical pregnancy and live birth rates (5). This correlation was not dependent on the patients' age nor the quality of the transferred embryo, which are known confounders for successful implantation.

Numerous therapies have since been employed to induce the receptivity of the endometrium and thus to improve implantation and pregnancy rates utilizing IVF.

For example, the 'Endometrial scratch' is one of the procedures through which multiple endometrial biopsies

Received 13 April 2018, Accepted 21 June 2018, Available online 11 July 2018

${ }^{1}$ Department of Obstetrics and Gynaecology, WITS, Johannesburg, South Africa. ${ }^{2}$ BioART Fertility Centre, Gynaecology and Infertility, Johannesburg, South Africa.

*Corresponding Author: Tasneem Mohamed, Email: tm.mohamed@yahoo.com 
are taken.

It is hypothesised that this procedure may enhance endometrial receptivity by increasing the production of cytokines and growth factors and thus promoting the decidualisation of the endometrium and embryo implantation. This 'local injury' to the endometrium may also allow for synchronization of the endometrium with the development of the embryo (6).

\section{Studies on the Use of G-CSF With IVF Therapy}

The use of G-CSF has variable effects on endometrial thickness and pregnancy outcomes.

As a pioneer researcher in this field, Gleicher et al showed that the intrauterine perfusion of G-CSF in women with a thin endometrium $(<7 \mathrm{~mm})$ resulted in the successful expansion of endometrium to a minimal thickness (>7 mm) within 48 hours after its infusion (7). In another study, Gleicher et al demonstrated an ongoing clinical pregnancy rate of $19.1 \%$ (8).

Similarly, Eftekhar et al evaluated the influence of G-CSF on improving endometrial thickness and pregnancy rates. The study group included 68 patients with a thin endometrium $(<7 \mathrm{~mm})$. Although the results of the study failed to show that G-CSF improved endometrial thickness, it was found that the use of G-CSF was associated with improved chemical and clinical pregnancy rates. The result, however, was not statistically significant (9). The above-mentioned researchers believed that G-CSF provides both chemical and mechanical stimulus, which may induce the secretion of cytokines. This endocrine-paracrine pathway possibly contributes to embryo implantation and pregnancy (9).

Furthermore, Kunicki et al investigated the influence of G-CSF on women undergoing IVF who had treatmentresistant thin endometrium $(<7 \mathrm{~mm})$. They divided the patients into two groups based on whether they conceived or not and found a clinical pregnancy rate of $18.9 \%$. Then, they assessed the change in endometrial thickness experienced by the groups and found a significant endometrial expansion in both groups (10).

The problem with failed IVF is not only psychologically traumatic for the concerned couple but also has huge financial implications.

\section{Objectives of the Study}

Transcervical intrauterine infusion of G-CSF is used at a fertility centre in Johannesburg for managing women with previously failed IVF. The purpose of the study was to describe the effects of the transcervical intrauterine infusion of G-CSF, as an adjunct to IVF, between January 2015 and August 2015. In other words, the objectives of the study were twofold:

1. To describe the effect of transvaginal intrauterine instillation of G-CSF, in adjunct to IVF treatment, on pregnancy rates;

2. To explore the effect of transvaginal infusion of
G-CSF on endometrial thickness.

\section{Materials and Methods}

Study Design

This study was of a retrospective cross-sectional type.

Study Population

The population of the study included a specific subgroup of women with at least two previously failed attempts at pregnancy with IVF. All women who were treated with G-CSF, as an adjunct to IVF, over the period formed part of the study.

Technique of Transvaginal G-CSF Infusion

The woman was placed in the lithotomy position and her vulva and vagina were cleansed with a disinfectant solution (savlon-cetrimide $3.0 \% \mathrm{w} / \mathrm{v}$ and chlorhexidine gluconate $0.3 \% \mathrm{w} / \mathrm{v}$ ), followed by inserting a Cusco speculum into the vagina in order to visualize the cervix. The cervix was then swabbed with a disinfectant solution and a Tomcat catheter was inserted into the mid-cavity of the uterus through the cervical canal. One ampoule of G-CSF (Neupogen ${ }^{\circledR}$, filgrastim), at room temperature, was drawn up into a $2 \mathrm{~mL}$ syringe and attached to the catheter. Next, the G-CSF was injected without using flush, followed by withdrawing the catheter and removing the speculum. Finally, the patient was left to lie supine for 10-15 minutes.

\section{Data Management}

Data were collected retrospectively and then exported to Stata $14.1^{\circledR}$ (StataCorp, 4905 Lakeway Drive, College Station, Texas 77845 USA) for analysis. Categorical and continuous variables were described using frequencies and percentages, as well as means (SD) and medians (interquartile range), respectively. Then, the results were compared using the chi-squared or Fisher exact tests. Continuous variables were compared using the Student's $t$ test or the Wilcoxon Mann-Whitney test. A difference was noted to be statistically significant when $P<0.05$.

\section{Explanatory Variables}

Several explanatory variables were utilized in this study such as age, parity, gravidity, previous obstetric history, the cause of infertility, previous fertility treatments, the employed IVF protocol, donor oocytes, embryo grading, and cryopreservation.

In addition, four different IVF protocols were used in this study as follows.

1. Long protocol (midluteal GnRHa downregulation protocol);

2. Step-up protocol (agonist antagonist protocol);

3. Frozen embryo transfer protocol (natural cycle/ hormonally manipulated cycle);

4. Oral ovulation induction protocol (clomiphene/ clomiphene and letrozole combination).

The embryos were graded on the third day of culturing 
according to the appearance of the embryo under the microscope using Veeck's day 3 scoring criteria. Further, the cryopreservation method applied was the rapid cooling 'vitrification' method.

Outcome Variables

The main outcome measure was the confirmation of pregnancy. The pregnancy result was initially determined 'biochemically' by serum B-HCG processed at Lancet laboratory on day 10 post-embryo transfer. If the result was positive, the patient was then examined after two weeks for ultrasound in order to confirm an ongoing pregnancy (i.e., clinical pregnancy). The secondary outcome measure encompassed the measurement of endometrial thickness post-G-CSF instillation compared with pre-G-CSF instillation.

Endometrium pre and post G-CSF was measured on the day of "triggering" and embryo transfer, respectively. Transvaginal G-CSF instillation was performed 2-4 days pre-embryo transfer.

\section{Results}

Demographics

A total of 103 women underwent ART therapy with granulocyte G-CSF in the stipulated period and the medical notes of 49 cases were reviewed in this regard. The excluded women had either less than two previous failed IVFs or had G-CSF infusion with another form of ART other than IVF (e.g., ovulation induction and intrauterine insemination).

Furthermore, the women were within the age range between 28 and 51 and the mean age was 38.90 (SD \pm 6.11). The median parity, the median gravidity, and the mean paternal age were 0 (within the range of $0-2$ ), 1 (within the range of $0-4$ ), and 42.70 ( $\mathrm{SD} \pm 6.96$, within the range of 29-61).

\section{Fertility History}

The number of previous IVF treatments ranged between 2 and 10 and the median was 2 (interquartile range, IQR: $2-4)$.

\section{Endometrial Thickness}

The endometrial thickness pre-G-CSF treatment ranged between $1.1 \mathrm{~mm}$ and $12 \mathrm{~mm}$. Moreover, the mean thickness and the median were $7.53 \mathrm{~mm}(\mathrm{SD} \pm 2.69)$ and $7.5 \mathrm{~mm}$ (IQR 6.1-9.8), respectively.

Likewise, the endometrial thickness post G-CSF was within the range of $4.8 \mathrm{~mm}$ to $14 \mathrm{~mm}$. Additionally, the mean thickness and median were $9.11 \mathrm{~mm}(\mathrm{SD} \pm 2.12)$ and $8.8 \mathrm{~mm}$ (IQR 7.5-11), respectively.

The comparison of the mean endometrial thickness preand post-G-CSF using the paired $t$ test yielded a $P<0.001$, implying a statistically significant difference.

The box plot in Figure 1 graphically shows the comparison of the mean endometrial thickness pre-

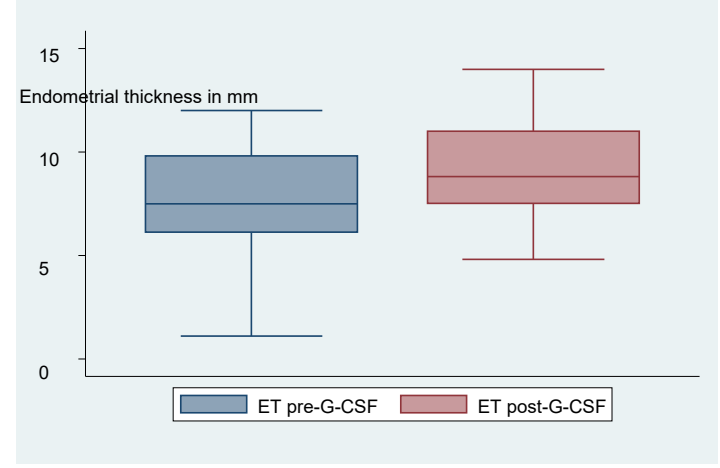

Figure 1. Box plot showing comparison of endometrial thickness pre and post granulocyte colony stimulating factor (G-CSF) in the women with "thin endometrium" (pre G-CSF $\leq 7 \mathrm{~mm}$ )

and post- G-CSF for specific women with a "thin endometrium" (pre G-CSF $\leq 7 \mathrm{~mm}$ ). This resulted in a $P=0.0004$, showing a statistically significant expansion of the endometrium post-G-CSF instillation.

Cycle Specific Data

A number of 11 out of 49 women used donor oocytes while one woman utilized both own and donor oocytes.

Additionally, the transferred embryos were either fresh embryos or thawed from previously cryopreserved embryos and two women had both transferred fresh and thawed embryos.

The mean and median number of the transferred fresh embryos was 2.83 ( $\mathrm{SD} \pm 1.14$ ) and 3 (IQR 2-4), respectively.

As regards the transferred frozen embryos, the mean and median number was $3.77(\mathrm{SD} \pm 1.31)$ and 3.5 (IQR 3-5), respectively.

For the purpose of analysis, the embryos were divided into good (either grade 1 or 2), intermediate (grade 3), and poor quality (grades 4 and 5). The majority (47) of cases had at least 1 good quality embryo.

Pregnancy Outcomes

In total, 17 women had a positive clinical pregnancy while 32 cases failed to fall pregnant.

Table 1 shows that younger women were more likely to fall pregnant and this was statistically significant. The comparison of the mean age indicated a $P<0.001$, affirming that the mean age between the two groups of women was statistically significant. There was no statistically significant difference in the other demographic factors. Similarly, no statistically significant difference was observed with respect to the causes of infertility or cycle specific factors (Tables 2 and 3, respectively).

\section{Discussion}

Pregnancy Outcomes

IVF treatment was successful in 17 out of 49 women, resulting in a pregnancy rate of $34.69 \%$. 
Table 1. Pregnancy Outcomes Related to Demographics and Fertility History

\begin{tabular}{lccc}
\hline & $\begin{array}{c}\text { Pregnancy } \\
\mathbf{n}=\mathbf{1 7}(\mathbf{3 4 . 6 9 \% )}\end{array}$ & $\begin{array}{c}\text { No Pregnancy } \\
\mathbf{n}=\mathbf{3 2}(\mathbf{6 5 . 3 1 \% )})\end{array}$ & $\boldsymbol{P}$ Value \\
\hline Demographics & & & \\
Age, mean (SD) & $34.94( \pm 1.15)$ & $41( \pm 5.76)$ & $<0.001$ \\
Age, median (IQR) & $34(31-37)$ & $40(36.5-46)$ & 0.001 \\
Age $<35$ & $10(62.5 \%)$ & $6(37.5 \%)$ & 0.004 \\
Age $<37$ & $13(45.52 \%)$ & $10(43.48 \%)$ & 0.003 \\
Parity, mean (SD) & $0.44( \pm 0.16)$ & $0.31( \pm 0.10)$ & 0.495 \\
Gravidity, mean (SD) & $1.06( \pm 0.023)$ & $1.03( \pm 0.21)$ & 0.925 \\
$\begin{array}{l}\text { Paternal age, mean } \\
\text { (SD) }\end{array}$ & $41.29( \pm 2.15)$ & $43.31( \pm 1.14)$ & 0.362 \\
$\begin{array}{l}\text { Fertility history } \\
\text { Number of previous }\end{array}$ & $3.18( \pm 0.40)$ & $3.13( \pm 0.33)$ & 0.922 \\
IVFs, mean (SD) & & & \\
\hline
\end{tabular}

Most studies to date have shown good pregnancy rates regarding the use of granulocyte colony-stimulating factor (G-CSF) (7-9).

In our study, age was the only statistically significant difference noted between those who either fell pregnant and those who failed to get pregnant. The mean age of these two groups was 35 and 41, respectively.

The achieved pregnancy rate was estimated at $45.68 \%$ at the fertility centre under investigation in 2014. Stratifying these data according to age, the pregnancy rates in those less than 35 years, between $35-37,38-40$, and $41-42$, and more than 42 years were $52.00 \%, 42.85 \%, 32.89 \%$, 33.33\%, and $46.42 \%$, respectively. By using G-CSF, pregnancy rates in the same age categories were equal to $62.29 \%, 44.44 \%$, $12.50 \%, 33.33 \%$, and $8.33 \%$, respectively. We are unable to make direct comparisons with regard to these results since the 2014 rates are the overall rates for the year not taking into account the woman's previous fertility treatments. In our study, the women undergoing G-CSF treatment were a particular subset in that they already had failed two or more previous IVF treatments.

Table 4 represents a comparison between the age of pregnancy rates achieved at the fertility centre under investigation in 2014 and the United States national 2014 rates as displayed in the summary report on the Society for Assisted Reproductive Technology website (11).

Table 2. Pregnancy Outcomes Related to Cause of Infertility

\begin{tabular}{|c|c|c|c|}
\hline & $\begin{array}{c}\text { Pregnancy } \\
\mathrm{n}=17(34.69 \%)\end{array}$ & $\begin{array}{l}\text { No Pregnancy } \\
n=32(65.31 \%)\end{array}$ & $P$-value \\
\hline \multicolumn{4}{|l|}{ Female causes of infertility } \\
\hline Polycystic ovarian syndrome, $n=7$ (14.29\%) & $1(14.29 \%)$ & $6(85.71 \%)$ & 0.397 \\
\hline Adenomyosis, $\mathrm{n}=5$ (10.2\%) & $1(20 \%)$ & $4(80 \%)$ & 0.646 \\
\hline Endometriosis, $\mathrm{n}=11$ (22.45\%) & $3(27.27 \%)$ & $8(72.73 \%)$ & 0.725 \\
\hline Fibroids, $n=6$ (12.24\%) & $1(16.67 \%)$ & $5(83.33 \%)$ & 0.650 \\
\hline Pelvic structural, $n=3(6.12 \%)$ & $0(0 \%)$ & $3(100 \%)$ & 0.542 \\
\hline Tubal, $n=14(28.7 \%)$ & $6(42.86 \%)$ & $8(5.14 \%)$ & 0.516 \\
\hline Ovulatory dysfunction, $\mathrm{n}=4(8.16 \%)$ & $0(0 \%)$ & $4(100 \%)$ & 0.284 \\
\hline Reduced ovarian reserve, $n=19$ (38.78\%) & $6(31.58 \%)$ & $13(68.42 \%)$ & 0.767 \\
\hline Chronic endometritis, $n=2$ (4.08\%) & $0(0 \%)$ & $2(100 \%)$ & 0.537 \\
\hline \multicolumn{4}{|l|}{ Male causes of infertility } \\
\hline Teratozoospermia, n=10 (20.41\%) & $3(30 \%)$ & $7(70 \%)$ & 1.000 \\
\hline Azoospermia, $n=2$ (4.08\%) & $1(50 \%)$ & $1(50 \%)$ & 1.000 \\
\hline Oligospermia, $n=4$ (8.16\%) & $0(0 \%)$ & $4(100 \%)$ & 0.284 \\
\hline Decreased motility, $n=2$ (4.08\%) & $0(0 \%)$ & $2(100 \%)$ & 0.537 \\
\hline
\end{tabular}

Table 3. Pregnancy Outcome Related to Cycle Specific Data

\begin{tabular}{|c|c|c|c|}
\hline All women & $\begin{array}{c}\text { Pregnancy } \\
n=17(34.69 \%)\end{array}$ & $\begin{array}{l}\text { No Pregnancy } \\
n=32(65.31 \%)\end{array}$ & $P$ Value \\
\hline Number of embryos transferred (Fresh/thawed) -mean (SD) & $3.82( \pm 1.29)$ & $3.13( \pm 3.13)$ & 0.081 \\
\hline ET pre-G-CSF - mean (SD) & $7.6( \pm 0.72)$ & $7.49( \pm 0.46)$ & 0.891 \\
\hline \multirow[t]{2}{*}{ ET post-G-CSF - mean (SD) } & $9.14( \pm 0.57)$ & $9.10( \pm 0.44)$ & 0.954 \\
\hline & $n=13(34.21 \%)$ & $n=25(65.79 \%)$ & \\
\hline ET expansion (Post G-CSF - Pre G-CSF) & $1.76( \pm 2.61)$ & $1.70( \pm 1.58)$ & 0.933 \\
\hline Women with ET pre-G-CSF $\leq 7 \mathrm{~mm}$ & $n=6(31.58 \%)$ & $n=13(68.42 \%)$ & \\
\hline ET pre-G-CSF - mean (SD) & $5.48( \pm 2.20)$ & $5.68( \pm 1.20)$ & 0.147 \\
\hline ET post-G-CSF - mean (SD) & $8.03( \pm 1.54)$ & $8.05( \pm 2.11)$ & $<0.001$ \\
\hline ET expansion (Post G-CSF - Pre G-CSF) & $2.55( \pm 3.64)$ & $2.36( \pm 1.87)$ & 0.882 \\
\hline
\end{tabular}

ET: Endometrial thickness; SD: Standard deviation; G-CSF: Granulocyte colony-stimulating factor. 


\begin{tabular}{lcc}
\multicolumn{3}{l}{ Table } \\
\hline Age & BioART 2014 & SART National Summary Report 2014 (11) \\
\hline$<35$ & 52.00 & 31.90 \\
$35-37$ & 42.85 & 26.50 \\
$38-40$ & 32.89 & 18.30 \\
$41-42$ & 33.33 & 10.50 \\
$>42$ & 46.42 & 3.60 \\
\hline
\end{tabular}

SART: Society for Assisted Reproductive Technology.

Based on the analysis, the pregnancy rates with G-CSF adjunctive therapy are higher than the previously obtained rate at BioART and is approximately double the US national rates in the groups aged $<35$ years and $35-37$ years. However, the true difference can only be determined using a randomized controlled trial (RCT).

\section{Endometrial Thickness}

Some studies showed that G-CSF has an effect on endometrial expansion while others denied such an effect (12). As part of the second objective of this study, we attempted to describe the effect of G-CSF on our study population although a thin endometrium was not a criterion for the study or for the use of G-CSF.

In our study sample, the overall expansion in endometrial thickness post G-CSF was found to be statistically significant.

However, a comparison of the mean endometrial thickness pre- and post- G-CSF between pregnant and non-pregnant women indicated no statistically significant difference between the two groups. ( $P=0.891$ and $P=0.954$, respectively)

Twenty-two out of 49 women had a pre-G-CSF endometrial thickness of $\leq 7 \mathrm{~mm}$. Therefore, these 22 women were particularly and carefully compared in terms of endometrial expansion post G-CSF. The comparison of the means of pre- and post-G-CSF endometrial thickness showed statistically significant endometrial expansion.

Accordingly, we found that younger women were more likely to fall pregnant and the rate of pregnancy decreased by $20 \%$ with every additional year of age. This is consistent with the results of other studies which have shown that age is an independent predictor of IVF success (13-15).

The results of this study showed no statistically significant difference between the two groups when comparing the means for parity and gravidity $(P=0.5$ and $P=0.93$, respectively), which is in line with the findings of Tsafrir et al, which demonstrated that previous pregnancy had no influence on the success of IVF treatment. Similarly, Rabinson et al, evaluating the influence of gravidity on IVF results, found that it has no effect on the likelihood of achieving pregnancy through IVF (16).

\section{Number of Previous IVF Cycles}

The mean number of previous IVF treatments in our sample was 3.14. The results of our study revealed no association between the number of previous IVF cycles and the pregnancy outcome $(P=0.922)$.

Cycle Specific Data

Fresh vs. Frozen Embryos

There is still much debate on whether the transfer of fresh embryo vs. cryopreserved/thawed embryo is superior or vice versa. Based on the findings of the current study, no relationship was found between the type of embryo and the pregnancy outcome $(P=1)$.

Other studies specifically advocated the cases with polycystic ovary syndrome, elective cryopreservation, and thawed embryo transfer in a subsequent cycle (17). In a cohort study, Roque et al compared IVF outcomes between fresh embryo transfer and frozen-thawed transfer (i.e., "freeze-all policy") and concluded that the outcomes were superior in the freeze-all policy group compared with the fresh embryo transfer group (18).

\section{Donor Oocytes vs Own Oocytes}

Patients who choose to use donor oocytes have severely diminished ovarian reserve and are unable to produce enough oocytes or the quality of their own oocytes is inadequate.

Our study demonstrated that there was an increased likelihood of pregnancy when using donor oocytes (odds ratio $=2.45,95 \% \mathrm{CI}=0.59-10.2$ ), but we failed to show any statistically significant difference between using either type of oocyte and the pregnancy outcome $(P=0.27)$.

\section{Number of Transferred Embryos}

In our study, the average number of transferred embryos was $3.37(\mathrm{SD} \pm 1.33)$ and ranged between 1 and 7 although no statistically significant relationship was observed between the number of embryos that were transferred and the pregnancy outcome $(P=0.081)$.

The likelihood of a successful pregnancy is hoped to increase by increasing the number of transferred embryos in an IVF cycle. Coughlan et al, in their review article on recurrent implantation failure, indicated that the probability of a single embryo to implant was approximately $30 \%$, hence, the probability of its failure for implantation was nearly $70 \%$. However, this probability reached $49 \%$ $\left(0.70^{2}=0.49\right)$ following the transfer of 2 embryos. Similarly, the transfer of $3,4,5$, or 6 embryos reduces the probability of implantation failure of all embryos by $34 \%, 24 \%$, $17 \%$, and $12 \%$, respectively (19). However, the transfer of multiple embryos has its downfall, along with the increased risk of multiple pregnancies and its associated pregnancy-related and perinatal complications.

\section{Embryo Grade}

The probability of failed implantation with a poor quality embryo is much higher than that with a good quality embryo.

A total of 47 out of 49 patients had at least one good 
quality embryo (grade 1 or 2). Of the other two remaining patients, 1 had only one grade 3 embryo and the other had three grade 5 embryos and both of them failed to fall pregnant.

As most of the patients in the two groups had good quality embryos, no further analysis was performed for comparison between the two groups.

Relation to the Cause of Infertility

Our study showed a similar frequency of the different causes of infertility as other studies although no statistically significant difference was found regarding the pregnancy outcome for any of the causes of infertility $(P>0.05)$.

To the best of our knowledge, this is the first study to describe the pregnancy rates with the use of G-CSF, as an adjunct to IVF, in the treatment of infertility in South Africa. It is unique in that all the included woman previously failed two more IVF cycles and not all of them had a thin endometrium. Therefore, this study adds to the expanding pool of research with respect to the various effects of G-CSF and its use in reproductive health.

\section{Strengths and Limitations}

The use of G-CSF instillation technique is the strong point of the present study and thus the study is reproducible.

Contrarily, this study has some limitations as follows:

1. The measure of pregnancy outcome was based on the result of the pregnancy test being either positive or negative. The overall success of the treatment, however, can only be determined by looking at the live birth rate. Unfortunately, patients were only followed up until 10 weeks of gestation. Therefore information regarding live births was unavailable.

2. This was a retrospective cross-sectional study thus some information was missing.

3. The study failed to take into account additional variables such as body mass index and the duration of infertility, which may affect the outcomes of IVF treatment.

4. Finally, the study aimed not to highlight the differences between women who became pregnant and those who did not. Even, in the case of differences between the 2 groups, it was negligible since this study was not sufficiently powered to determine such differences.

\section{Conclusions}

In this study, the pregnancy rate was $34.69 \%$. Whilst we showed an increase in endometrial thickness we were unable to show any statistically significant association between endometrial expansion and pregnancy outcome as the study was underpowered to determine this.

There is a large body of research regarding the benefits of granulocyte colony-stimulating factor (G-CSF) in reproductive health. A recent study evaluated the subcutaneous use of G-CSF in women with repeated IVF failures and reported statistically significant improved implantation and clinical pregnancy rates (20).

This study is the evidence in favour of others who alluded to the fact that G-CSF may have other effects on the achievement of pregnancy other than just expanding the endometrium. The overall effect of the use of G-CSF with assisted reproductive technology seems promising.

\section{Clinical Recommendations}

Only an RCT is able to answer whether the pregnancy rate of $34.69 \%$ is indeed superior in women who had previously failed IVF procedures. We recommend future researchers to conduct an adequately powered well-structured RCT.

\section{Conflict of Interests}

Authors declare that they have no conflict of interests.

\section{Ethical Issues}

This study was approved and granted by the University of the Human Research and Ethics Committee of University of Witwatersrand (Clearance certificate no: M150961).

\section{Financial Support}

None.

\section{References}

1. Demetri GD, Griffin JD. Granulocyte colony-stimulating factor and its receptor. Blood 1991;78:2791-2808.

2. Allahbadia G. The unfinished story of granulocyte colonystimulating factor in assisted reproductive technology. IVF Lite 2014;1(3):139-145.

3. Ziebe S, Loft A, Povlsen BB, et al. A randomized clinical trial to evaluate the effect of granulocyte-macrophage colonystimulating factor (GM-CSF) in embryo culture medium for in vitro fertilization. Fertil Steril. 2013;99(6):1600-9. doi: 10.1016/j.fertnstert.2012.12.043.

4. Al-Ghamdi A, Cokun S, Al-Hassan S, Al-Rejjal R, Awartani $\mathrm{K}$. The correlation between endometrial thickness and outcome of in vitro fertilization and embryo transfer (IVFET) outcome. Reprod Biol Endocrinol. 2008 Sep 2;6:37. doi: 10.1186/1477-7827-6-37.

5. Richter KS, Bugge KR, Bromer JG, Levy MJ. Relationship between endometrial thickness and embryo implantation, based on 1,294 cycles of in vitro fertilization with transfer of two blastocyst-stage embryos. Fertil Steril. 2007;87(1):5359.

6. Zhou L, Li R, Wang R, Huang H, Zhong K. Local injury to the endometrium in controlled ovarian hyperstimulation cycles improve implantation rates. Fertil Steril. 2008;89(5):11661176.

7. Gleicher N, Vidali A, Barad DH. Successful treatment of unresponsive thin endometrium. Fertil Steril. 2011;95(6)2123e13-e17.

8. Gleicher N, Kim A, Michaeli T, et al. A pilot cohort study of granulocyte colony-stimulating factor in the treatment of unresponsive thin endometrium resistant to standard therapies. Hum Reprod. 2013;28(1):172-177. doi: 10.1093/ humrep/des370.

9. Eftekhar M, Sayadi M, Arabjahvani F. Transvaginal 
perfusion of G-CSF for infertile women with thin endometrium in frozen ET program: A non-randomized clinical trial. Iran J Reprod Med. 2014;12(10):661-666.

10. Kunicki M, Lukaszuk K, Woclawk-Potocka I, Liss J, Kulwikowska P, Szczyptanska J. Evaluation of granulocyte colony-stimulating factor effects on treatment-resistant thin endometrium in women undergoing in vitro fertilization. Biomed Res Int 2014;2014:913235.

11. National summary report. SART database. 2013 [cited 2016 June 24]. https://www.sartcorsonline.com/rptCSR_ PublicMultYear.aspx?ClinicPKID $=0$.

12. Kasius A, Smit JG, Torrance HL, et al. Endometrial thickness and pregnancy rates after IVF: a systematic review and meta-analysis. Hum Reprod Update. 2014;20(4):530-541.

13. Tsafrir A, Simon A, Revel A, Reubinoff B, Lewin A, Laufer N. Retrospective analysis of 1217 IVF cycles in women aged 40 years and older. Reprod Biomed Online. 2007;14(3):348355.

14. Dhillon RK, McLernon DJ, Smith PP, et al. Predicting the chance of live birth for women undergoing IVF: a novel pretreatment counselling tool. Hum Reprod. 2016;31(1):8492.
15. Revelli A, Biasoni V, Gennarelli G, Canosa S, Dalmasso P, Benedetto C. IVF results in patients with very low serum $\mathrm{AMH}$ are significantly affected by chronological age. J Assist Reprod Genet. 2016;33(5):603-609. doi: 10.1007/ s10815-016-0675-7.

16. Rabinson J, Bar-Hava I, Meltcer S, Zohav E, Anteby E, Orvieto R. Does gravidity influence the success of in vitro fertilization- embryo transfer cycles? Gynecol Endocrinol. 2006;22(4):175-8. doi:10.1080/09513590600630512

17. Chen J, Shi Y, Sun Y, et al. Fresh versus frozen embryos for infertility in the polycystic ovary syndrome. N Engl J Med. 2016 Aug 11;375(6):523-33. doi: 10.1056/NEJMoa1513873.

18. Roque M, Valle M, Guimarães F, Sampaio M, Geber S. Freeze-all policy: fresh vs. frozen-thawed embryo transfer. Fertil Steril. 2015;103(5):1190-1193.

19. Coughlan C, Ledger W, Wang Q, et al. Recurrent implantation failure: definition and management. Reprod Biomed Online. 2014;28:14-38.

20. Aleyasin A, Abediasl Z, Nazari A, Sheikh M. Granulocyte colony-stimulating factor in repeated IVF failure, a randomized trial. Reproduction 2016;151(6):637-642. doi: 10.1530/REP-16-0046.

(c) 2019 The Author (s); This is an open-access article distributed under the terms of the Creative Commons Attribution License (http://creativecommons.org/licenses/by/4.0), which permits unrestricted use, distribution, and reproduction in any medium, provided the original work is properly cited. 